\title{
A New Measure of Quantum Starlike Functions Connected with Julia Functions
}

\author{
Samir B. Hadid $\mathbb{D},^{1,2}$ Rabha W. Ibrahim $\mathbb{D}^{3},^{3}$ and Shaher Momani $\mathbb{D}^{1,2,4}$ \\ ${ }^{1}$ Department of Mathematics and Sciences, College of Humanities and Sciences, Ajman University, Ajman, UAE \\ ${ }^{2}$ Nonlinear Dynamics Research Center (NDRC), Ajman University, Ajman, UAE \\ ${ }^{3}$ IEEE: 94086547, Perrysburg, Ohio, USA \\ ${ }^{4}$ Department of Mathematics, Faculty of Science, University of Jordan, Amman 11942, Jordan \\ Correspondence should be addressed to Rabha W. Ibrahim; rabhaibrahim@yahoo.com
}

Received 9 December 2021; Accepted 8 January 2022; Published 30 January 2022

Academic Editor: Sarfraz Nawaz Malik

Copyright (c) 2022 Samir B. Hadid et al. This is an open access article distributed under the Creative Commons Attribution License, which permits unrestricted use, distribution, and reproduction in any medium, provided the original work is properly cited.

\begin{abstract}
In a complex domain, the investigation of the quantum differential subordinations for starlike functions is newly considered by few research studies. In this note, we arrange a set of necessary conditions utilizing the concept of the quantum differential subordinations for starlike functions related to the set of parametric Julia functions. Our method is based on the usage of quantum Jack lemma, where this lemma is generalized recently by the quantum derivative (Jackson calculus). We illustrate a starlike formula dominated by different types of Julia functions. The sufficient conditions are computed in the quantum and the Julia fractional parameters. We indicate a relationship between these two parameters.
\end{abstract}

\section{Introduction}

The notion of differential subordination and superordination (DSS) shows a dynamic model in the investigation of geometric possessions of holomorphic functions in the open unit disk. Lindelof first presented it, while Littlewood [1] did the extraordinary exertion in this area of study. Numerous investigators added information in the application of DSS. Antiquity and the improvement of mechanisms in the area connected with DSS are concisely designated and incorporated in the hardcover by Miller and Mocanu [2]. The main growth in the area of derivative of DSS began by Miller et al. [3]. Generally, the concept is defined for univalent function $\omega$ by

$$
\varphi \prec \psi \Leftrightarrow \varphi(0)=\psi(0),
$$

and $\varphi(\omega) \subset \psi(\omega)$. In general, if there is a function with the properties $\omega(0)=0,|\omega(\xi)|<|\xi|$, satisfying $\varphi(\xi)=\psi(\omega(\xi))$, then

$$
\varphi(\xi) \prec \psi(\xi)
$$

where $\xi \in \sqcup:=\{\xi \in \mathbb{C}:|\xi|<1\}$.

Ismail et al. [4] presented a class of complex functions for each fractional number $q, 0<q<1$ as the class of analytic functions $\varphi$ on the open unit disk $(\sqcup), \varphi(0)=0, \varphi^{\prime}(0)=1$, and $|\varphi(q \xi)| \leq|\varphi(\xi)|$ on $\sqcup$. This class is investigated, as well as the links between it and other analytic function classes. Agrawal and Sahoo [5] extended this notion by suggesting the $q$-starlike functions family in a logical order. Srivastava et al. [6] explored the link between the Janowski functions and several known types of $q$-starlike functions. The Janowski functions are a novel subclass of $q$-starlike functions that they introduced and presented. Recent investigations can be located in works by Mahmood et al. [7] and Ul-Haq et al. [8].

Parametric Julia functions are usually utilized to determine the upper bound solutions of different types of differential equations of a complex variable [4-11]. In the recent study, we shall extend this concept applying the quantum calculus (Jackson calculus) and employ it to define special classes of analytic function types normalized analytically in the open unit disk $(\varphi(0)=0, \varphi \prime(0)=1)$ and 
dominated by different kinds of the parametric Julia functions. Our method is based on the quantum Jack lemma.

\section{Quantum Starlike Formula}

The effort of Ma and Minda [12] in this area of studies is not minor as they considered the normalized analytic function $p(0)=1$ and the condition of a positive real part $\mathfrak{R}\left(p^{\prime}(\xi)\right)>0$. They have formulated the famous subclasses for starlike and convex functions, as follows, respectively:

$$
\begin{aligned}
\mathcal{S}^{*}(p) & =\left\{\varphi \in \Delta: \frac{\xi \varphi \prime(\xi)}{\varphi(\xi)}<p(\xi), \xi \in \sqcup\right\}, \\
\mathscr{C}(p) & =\left\{\varphi \in \Delta: \frac{\xi \varphi^{\prime \prime}(\xi)}{\varphi^{\prime}(\xi)}+1<p(\xi), \xi \in \sqcup\right\},
\end{aligned}
$$

where $\Delta$ indicates the class of normalized function $\varphi(0)=0=\varphi \prime(0)-1$.

Quantum calculus (QC) is the novel part of mathematical analysis and its applications and is correspondingly significant for its appearances, both in physics and in mathematics as well. Jackson $[13,14]$ formulated the functions of q-differentiation and $q$-integration and decorated their meanings for the first stage. Later, Ismail et al. [4] contributed the indication of q-calculus in geometric function theory.

Nowadays, different classes of Ma and Minda are suggested and developed, using QC by researchers. For instant, Seoudy and Aouf [15] introduced subclass of quantum starlike functions involving q-derivative. Recently, Zainab et al. [16] presented a sufficient condition for $q$-starlikeness using a special curve. In addition, different differential and integral operators are generalized utilizing QC [17-20].

Definition 1. Jackson derivative is indicated in the following difference operator:

$$
\left(\partial_{q} h\right)(\xi)=\frac{h(\xi)-h(q \xi)}{\xi(1-q)}, \quad q \in(0,1),
$$

such that

$$
\partial_{q}\left(\xi^{v}\right)=\left(\frac{1-q^{v}}{1-q}\right) \xi^{\nu-1}
$$

Moreover, Maclaurin's series representation takes the sum

$$
\left(\partial_{q} h\right)(\xi)=\sum_{\ell=0}^{\infty} h_{\ell}[\ell]_{q} \xi^{\ell-1}
$$

where

$$
[\ell]_{q}:=\frac{1-q^{\ell}}{1-q} .
$$

Note that

$$
\lim _{q \longrightarrow 1^{-}}\left(\partial_{q} h\right)(\xi)=h \prime(\xi) .
$$

The multiplication rule takes the following formula:

$$
\begin{aligned}
\partial_{q}(f(\xi) g(\xi)) & =g(\xi) \partial_{q} f(\xi)+f(q \xi) \partial_{q} g(\xi) \\
& =g(q \xi) \partial_{q} f(\xi)+f(\xi) \partial_{q} g(\xi) .
\end{aligned}
$$

We proceed to define our $q$-starlike class using the $q$-parametric Julia functions and connecting with the subclass of normalized functions in $\sqcup$ (Figure 1):

$$
\begin{gathered}
J_{1}^{(\beta)}(\xi)=1+\xi-\beta \xi^{3} \\
J_{2}^{(\beta)}(\xi)=\left(1+\xi-\beta \xi^{2}\right)^{2}, \\
J_{3}^{(\beta)}(\xi)=1+\xi-\beta \xi^{2}, \\
(\beta \in \mathbb{C}, \xi \in \sqcup)
\end{gathered}
$$

Definition 2. For a normalized function $\varphi(\xi) \in \Delta$ of the formula

$$
\varphi(\xi)=\xi+\sum_{n=2}^{\infty} \varphi_{n} \xi^{n}, \quad \xi \in \sqcup,
$$

the $q$-starlike is defined by the subordination formula:

$$
\begin{array}{r}
\frac{\xi\left(\partial_{q} \varphi\right)(\xi)}{\varphi(\xi)}<J_{i}^{(\beta)}(\xi) \\
(i=1,2,3, q \in(0,1), \beta \in \mathbb{C}) .
\end{array}
$$

We denote the subclass of these functions by $\Delta_{q}^{(\beta)}$, where

$$
\left(\partial_{q} \varphi\right)=1+\sum_{n=2}^{\infty} \varphi_{n}\left(\frac{1-q^{n}}{1-q}\right) \xi^{n} .
$$

Moreover, a function $\varphi \in \Delta$ is called $q$-bounded turning if it satisfies the inequality

$$
\partial_{q} \varphi(\xi) \prec J_{i}^{(\beta)}(\xi) .
$$

We denote this class by $\mathbb{B}_{q}^{(\beta)}$.

We aim to find the range of $\beta$ in terms of $q$ satisfying the inequality (14). For this purpose, we need the following result.

Lemma 1 (see [21]). Let $₫$ be analytic in $\sqcup$, such that $\varpi(0)=0$. Then, the upper value of $₫$ on the circle $|\xi|=1$ at the point $\xi_{0}=r e^{i \theta}, \theta \in[-\pi, \pi], q \in(0,1)$, is

$$
\xi_{0}\left(\partial_{q} \varpi\left(\xi_{0}\right)\right)=\mu \varpi\left(\xi_{0}\right), \quad \mu \geq 1 .
$$

\section{Results}

In this section, we shall illustrate the sufficient conditions on functions $\varphi \in \Delta$ to be in $\Delta_{q}^{(\beta)}$. 

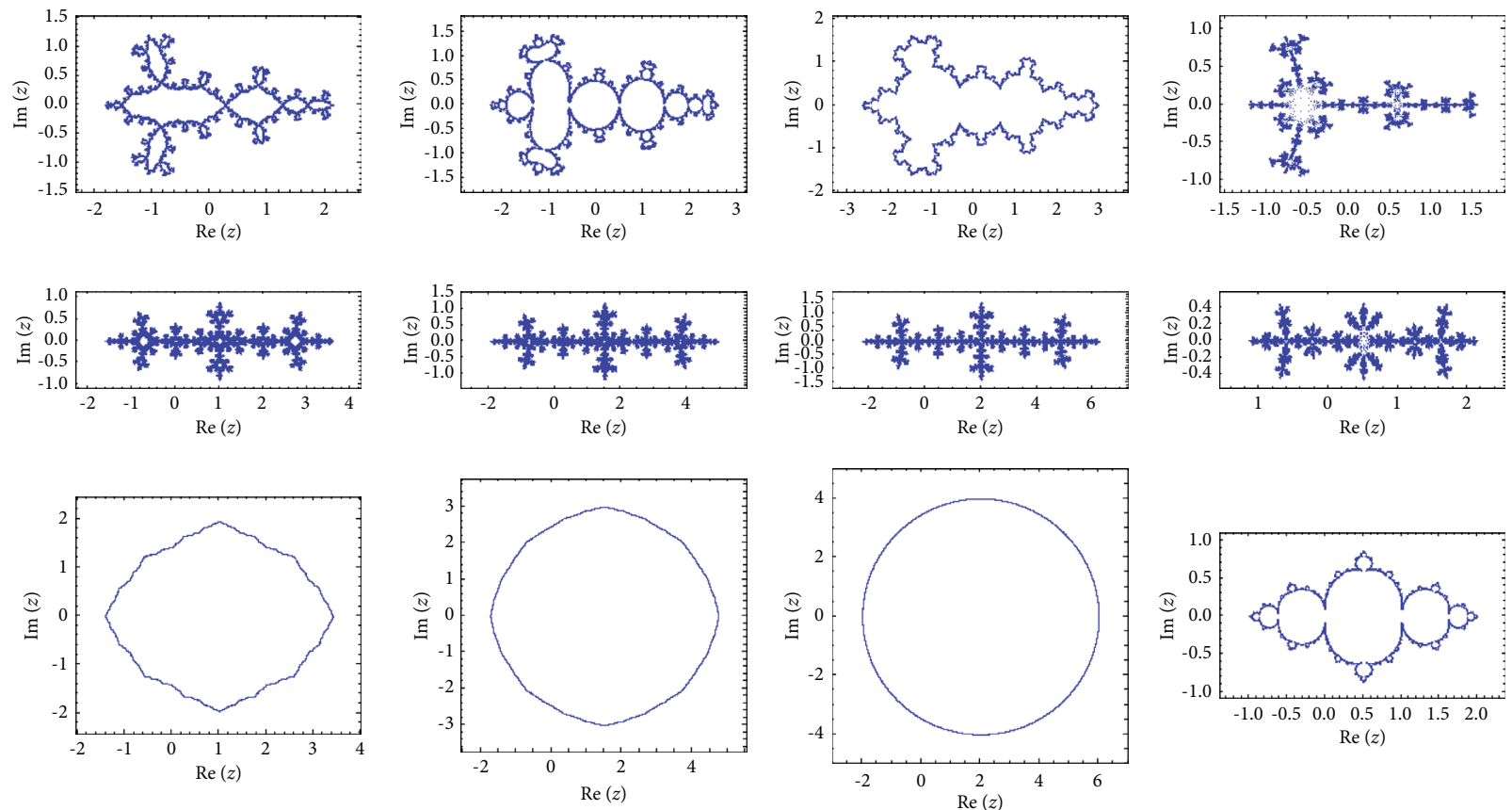

Figure 1: Plot of $J_{i}^{(\beta)}, i=1,2,3$ for $\beta=1 / 2,1 / 3,1 / 4$ and $\beta=1$, respectively. The plot is connected when $\beta \in(0,1]$; otherwise, it is disconnected.

Theorem 1. Let the function $\rho \in \sqcup$, such that $\rho(0)=1$ and

$$
1+\xi\left(\partial_{q} \rho(\xi)\right) \prec \sqrt{1+\xi}, \quad \xi \sqcup .
$$

If one of the cases

$$
\beta \neq \frac{q+1}{q^{2}-q+1}, \beta<\frac{q+1+\sqrt{2}}{q^{2}-q+1}, \beta>\frac{q+1-\sqrt{2}}{q^{2}-q+1}, \quad q \in(0,1),
$$

holds, then

$$
\rho(\xi) \prec J_{1}^{(\beta)}(\xi)=1+\xi-\beta \xi^{3} .
$$

Proof. Define a function $\varrho$ as follows:

$$
\varrho(\xi):=1+\xi\left(\partial_{q} \rho(\xi)\right)
$$

By the assumption (18) and the definition of the subordination, we have

$$
1+\xi\left(\partial_{q} \rho(\xi)\right)=\sqrt{1+\Phi(\xi)}, \quad \varpi(0)=0,|\bowtie(\xi)| \leq|\xi|<1
$$

which leads to

$$
\varpi(\xi)=\varrho^{2}(\xi)-1
$$

We aim to show that $\left|\varrho^{2}(\xi)-1\right|<1$ for some values $\xi_{0} \in \sqcup$, such that

$$
\rho(\xi)=1+\omega(\xi)-\beta \omega^{3}(\xi) .
$$

Assume not; then, the above conclusion implies that

$$
\varrho(\xi)=1+\xi\left(\partial_{q}\left[1+\omega(\xi)-\beta \varpi^{3}(\xi)\right]\right) .
$$

By using the rules of Jackson derivative, we obtain

$\partial_{q} \omega^{2}(\xi)=\partial_{q} \omega(\xi)[\omega(\xi)+\varpi(q \xi)]$,

$\partial_{q} \varpi^{3}(\xi)=\partial_{q} \bowtie(\xi)\left[\omega^{2}(\xi)+\varpi(\xi) \bowtie(q \xi)+\varpi^{2}(q \xi)\right]$.

Consequently, we get

$$
\varrho(\xi)=1+W_{q}(\xi)\left(\xi \partial_{q} \bowtie(\xi)\right)
$$

where

$W_{q}(\xi):=\varpi(\xi)+\Phi(q \xi)-\beta\left(\varpi^{2}(\xi)+\omega(\xi) \omega(q \xi)+\Phi^{2}(q \xi)\right)$.

But

$$
\varpi(q \xi)=\omega(\xi)-(1-q) \xi\left(\partial_{q} \varpi(\xi)\right) .
$$

Hence, this yields

$$
W_{q}(\xi)=\varpi(\xi)[2-3 \beta \bowtie(\xi)]+\xi \partial_{q} \bowtie(\xi)\left[-(1-q)+(3-q) \Subset(\xi)-\beta(1-q)^{2} \xi \partial_{q} \bowtie(\xi)\right]
$$


Suppose that there exists a point $\xi_{0} \in \sqcup$, such that

$$
\begin{aligned}
& \max _{|\xi| \leq\left|\xi_{0}\right|}|\emptyset(\xi)|=\left|\varpi\left(\xi_{0}\right)\right|=1, \\
& \xi_{0}\left(\partial_{q} @\left(\xi_{0}\right)\right)=\mu \varpi\left(\xi_{0}\right), \quad \mu \geq 1 .
\end{aligned}
$$

$$
\begin{aligned}
\left|W_{q}\left(\xi_{0}\right)\right|= & \left|\bowtie(\xi)[2-3 \beta \bowtie(\xi)]+\xi \partial_{q} \varpi(\xi)\left[-(1-q)+\beta(3-q) \bowtie(\xi)-\beta(1-q)^{2} \xi \partial_{q} \varpi(\xi)\right]\right|_{\xi=\xi_{0}} \\
= & \left|e^{i \theta}\left[2-3 \beta e^{i \theta}\right]+\mu e^{i \theta}\left[-(1-q)+\beta(3-q) e^{i \theta}-\beta(1-q)^{2} \mu e^{i \theta}\right]\right| \\
& \geq \Re\left(e^{i \theta}\left[2-3 \beta e^{i \theta}\right]+\mu e^{i \theta}\left[-(1-q)+\beta(3-q) e^{i \theta}-\beta(1-q)^{2} \mu e^{i \theta}\right]\right) \\
= & \cos (\theta)[2-3 \beta \cos (\theta)]+\mu \cos (\theta)\left[-(1-q)+\beta(3-q) \cos (\theta)-\beta(1-q)^{2} \mu \cos (\theta)\right] \\
= & \beta\left[-3+\mu(3-q)-\mu(1-q)^{2}\right] \cos ^{2}(\theta)+[2-\mu(1-q)] \cos (\theta) \\
= & \beta\left[\mu\left(3-q-(1-q)^{2}\right)-3\right] \cos ^{2}(\theta)+[2-\mu(1-q)] \cos (\theta) .
\end{aligned}
$$

Accordingly, we conclude that

$$
\begin{aligned}
\left|\varrho(\xi)^{2}-1\right|_{\xi=\xi_{0}}= & \left|\left(1+W_{q}(\xi)\left(\xi \partial_{q} \bowtie(\xi)\right)\right)^{2}-1\right|_{\xi=\xi_{0}} \\
& \geq\left|\left(W_{q}\left(\xi_{0}\right)\left(\xi_{0} \partial_{q} \bowtie\left(\xi_{0}\right)\right)\right)^{2}-1\right| \\
= & \left|\left(\mu \beta\left[\mu\left(3-q-(1-q)^{2}\right)-3\right] \cos ^{3}(\theta)+\mu[2-\mu(1-q)] \cos ^{2}(\theta)\right)^{2}-1\right| \\
& \geq\left|\left(\beta\left[\left(3-q-(1-q)^{2}\right)-3\right]+[1+q]\right)^{2}-1\right| \geq 1,
\end{aligned}
$$

provided one of the following cases holds

$$
\Upsilon \leq-\sqrt{2}, \Upsilon=0, \Upsilon \geq \sqrt{2},
$$

where

$$
\Upsilon:=\beta\left[\left(3-q-(1-q)^{2}\right)-3\right]+[1+q] .
$$

Hence, we obtain one of the following arguments:

$$
\beta=\frac{q+1}{q^{2}-q+1}, \beta \geq \frac{q+1+\sqrt{2}}{q^{2}-q+1}, \beta \leq \frac{q+1-\sqrt{2}}{q^{2}-q+1},
$$

which are all contradict (19), that is

$$
\rho(\xi)<J_{1}^{(\beta)}(\xi)=1+\xi-\beta \xi^{3} .
$$

As a special case, we have the following result.

Corollary 1. Let $\varphi \in \Delta$ be satisfied the subordination:

$$
1+\xi\left(\partial_{q}\left(\frac{\xi \partial_{q} \varphi(\xi)}{\varphi(\xi)}\right)\right)<\sqrt{1+\xi}, \quad \xi \in \sqcup .
$$

If one of the cases in (21) is occurred, then $\varphi \in \Delta_{q}^{(\beta)}$.

Proof. Assume

$$
\rho(\xi)=\left(\frac{\xi \partial_{q} \varphi(\xi)}{\varphi(\xi)}\right) .
$$

Obviously, $\rho(0)=1$. Thus, in virtue of Theorem 1, we have $\varphi \in \Delta_{q}^{(\beta)}$.

Similarly, by assuming $\rho(\xi)=\partial_{q} \varphi(\xi), \varphi \in \Delta$, we have the following result.

Corollary 2. Let $\varphi \in \Delta$ be the satisfied subordination:

$$
1+\xi\left(\partial_{q} \varphi(\xi)\right)<\sqrt{1+\xi}, \quad \xi \in \sqcup .
$$

If one of the cases in (21) is occurred, then $\varphi \in \mathbb{B}_{q}^{(\beta)}$.

Theorem 2. Let the function $h \in \sqcup$, such that $h(0)=1$ and

$$
1+\xi\left(\partial_{q} h(\xi)\right)<\sqrt{1+\xi}, \quad \xi \in \sqcup,
$$

if one of the cases

$$
\beta \neq \frac{1}{q+1}, \beta \neq 2,
$$

where for $0.418341<q<1$, 


$$
\begin{gathered}
\frac{\left.\left(0.5\left(2 q+\sqrt{(2 q+3)^{2}-10.3784(q+1)}\right)+3\right)\right)}{q+1} \geq \beta \\
\geq \frac{\left(0.5\left(2 q-\sqrt{(2 q+3)^{2}-5.62159(q+1)}\right)+3\right)}{q+1},
\end{gathered}
$$

and for $0<q<0.418341$,

$$
\begin{aligned}
& \frac{\left(0.5\left(2 q+\sqrt{(2 q+3)^{2}-10.3784(q+1)}\right)+3\right)}{q+1} \geq \beta \\
& \geq \frac{\left(0.5\left(2 q-\sqrt{(2 q+3)^{2}-10.3784(q+1)}\right)+3\right)}{q+1},
\end{aligned}
$$

hold; then,

$$
h(\xi)<J_{2}^{(\beta)}(\xi)=\left(1+\xi-\beta \xi^{2}\right)^{2} .
$$

Proof. Define a function $p$ as follows:

$$
p(\xi):=1+\xi\left(\partial_{q} h(\xi)\right) .
$$

$$
1+\xi\left(\partial_{q} h(\xi)\right)=\sqrt{1+w(\xi)}, \quad w(0)=0,|w(\xi)| \leq|\xi|<1,
$$

which yields

$$
w(\xi)=p^{2}(\xi)-1 .
$$

We have to prove that

$$
|w(\xi)|=\left|p^{2}(\xi)-1\right|<1,
$$

for some values $\xi_{0} \in \sqcup$, such that

$$
h(\xi)=\left[1+w(\xi)-\beta w^{2}(\xi)\right]^{2} .
$$

Assume not; then, the above conclusion imposes

$$
p(\xi)=1+\xi\left(\partial_{q}\left[1+w(\xi)-\beta w^{2}(\xi)\right]^{2}\right) .
$$

By using the rules of Jackson derivative and the facts

$$
\begin{aligned}
w(q \xi) & =w(\xi)-(1-q) \xi \partial_{q} w(\xi), \\
\partial_{q} w^{2}(\xi) & =\partial_{q} w(\xi)\left[2 w(\xi)-(1-q) \xi \partial_{q} w(\xi)\right],
\end{aligned}
$$

we obtain

By the assumption (41) and the meninges of the subordination, we have

$$
\begin{aligned}
& \partial_{q}\left[1+w(\xi)-\beta w^{2}(\xi)\right]^{2}=\left[1+w(\xi)-\beta w^{2}(\xi)\right] \partial_{q}\left[1+w(\xi)-\beta w^{2}(\xi)\right] \\
& \quad+\left[1+w(q \xi)-\beta w^{2}(q \xi)\right] \partial_{q}\left[1+w(\xi)-\beta w^{2}(\xi)\right]=2\left[1+w(\xi)-\beta w^{2}(\xi)\right] \\
& \left(\partial_{q} w(\xi)-\beta \partial_{q} w(\xi)\left[2 w(\xi)-(1-q) \xi \partial_{q} w(\xi)\right]\right)=2\left[1+w(\xi)-\beta w^{2}(\xi)\right] \\
& \partial_{q} w(\xi)\left(1-\beta\left[2 w(\xi)-(1-q) \xi \partial_{q} w(\xi)\right]\right) .
\end{aligned}
$$

Consequently, we get

$$
p(\xi)=1+2 \xi\left[1+w(\xi)-\beta w^{2}(\xi)\right] \partial_{q} w(\xi)\left(1-\beta\left[2 w(\xi)-(1-q) \xi \partial_{q} w(\xi)\right]\right) .
$$


Suppose that there exists a point $\xi_{0} \in \sqcup$, such that

$$
\begin{aligned}
& \max _{|\xi| \leq\left|\xi_{0}\right|}|w(\xi)|=\left|w\left(\xi_{0}\right)\right|=1, \\
& \xi_{0}\left(\partial_{q} w\left(\xi_{0}\right)\right)=\mu w\left(\xi_{0}\right), \quad \mu \geq 1 .
\end{aligned}
$$

We aim to show that

$$
|w(\xi)|=\left|p^{2}(\xi)-1\right|<1
$$

Our method is based on Jack Lemma 1. Assume not. Then, by consuming $w\left(\xi_{0}\right)=e^{i \theta}$, we get

$$
\begin{aligned}
\left|p^{2}\left(\xi_{0}\right)-1\right|= & \left|\left(1+2 \xi\left[1+w(\xi)-\beta w^{2}(\xi)\right] \partial_{q} w(\xi)\left(1-\beta\left[2 w(\xi)-(1-q) \xi \partial_{q} w(\xi)\right]\right)\right)^{2}-1\right|_{\xi=\xi_{0}} \\
& \geq\left|4\left[1+e^{i \theta}-\beta e^{2 i \theta}\right]^{2}\left(\mu e^{i \theta}\left(1-\beta\left[2 e^{i \theta}-(1-q) \mu e^{i \theta}\right]\right)\right)^{2}-1\right| \\
& \geq \Re\left(4\left[1+e^{i \theta}-\beta e^{2 i \theta}\right]^{2}\left(\mu e^{i \theta}\left(1-\beta\left[2 e^{i \theta}-(1-q) \mu e^{i \theta}\right]\right)\right)^{2}-1\right) \\
& =4\left[1+\cos (\theta)-\beta \cos ^{2}(\theta)\right]^{2}(\mu \cos (\theta)(1-\beta[2 \cos (\theta)-(1-q) \mu \cos (\theta)]))^{2}-1 \geq 1 .
\end{aligned}
$$

Then, the solution when $\cos (\theta)=1$ of

$$
\left|4\left[1+\cos (\theta)-\beta \cos ^{2}(\theta)\right]^{2}(\mu \cos (\theta)(1-\beta[2 \cos (\theta)-(1-q) \mu \cos (\theta)]))^{2}-1\right| \geq 1
$$

brings one of the following cases:

$$
\curlywedge=0, \curlywedge \geq \sqrt{2}, \curlywedge \leq-\sqrt{2},
$$

where

$$
\text { ᄉ: }=4(2-\beta)^{2}(1-\beta(1+q))^{2} \text {. }
$$

Hence, we obtain one of the following arguments:

$$
\beta=\frac{1}{q+1}, \beta=2,
$$

and for $0.418341<q<1$,

$$
\begin{gathered}
\frac{\left(\left(0.52 q+\sqrt{(2 q+3)^{2}-10.3784(q+1)}\right)+3\right)}{q+1} \leq \beta \\
\leq \frac{\left(0.5\left(2 q-\sqrt{(2 q+3)^{2}-5.62159(q+1)}\right)+3\right)}{q+1} .
\end{gathered}
$$

Moreover, for $0<q<0.418341$, we have

$$
\begin{gathered}
\frac{\left(0.5\left(2 q+\sqrt{(2 q+3)^{2}-10.3784(q+1)}\right)+3\right)}{q+1} \leq \beta \\
\leq \frac{\left(0.5\left(2 q-\sqrt{(2 q+3)^{2}-10.3784(q+1)}\right)+3\right)}{q+1} .
\end{gathered}
$$

All the above inequalities contradict the assumptions of the theorem, which lead to

$$
h(\xi) \prec J_{2}^{(\beta)}(\xi)=\left(1+\xi-\beta \xi^{2}\right)^{2} .
$$

Corollary 3. Let $\varphi \in \Delta$ be the satisfied subordination:

$$
1+\xi\left(\frac{\xi\left(\partial_{q} \varphi(\xi)\right)}{\varphi(\xi)}\right) \prec \sqrt{1+\xi}
$$

If one of the assumptions of Theorem 2 is occurred, then $\varphi \in \Delta_{q}^{(\beta)}$.

Proof. Assume

$$
p(\xi)=\left(\frac{\xi \partial_{q} \varphi(\xi)}{\varphi(\xi)}\right) .
$$

Obviously, $p(0)=1$. Thus, according to Theorem 2, we get $\varphi \in \Delta_{q}^{(\beta)}$.

In the same manner of the above result, we obtain the next one when $p(\xi)=\partial_{q} \varphi(\xi), \varphi \in \Delta$.

Corollary 4. Let $\varphi \in \Delta$ be the satisfied subordination:

$$
1+\xi\left(\partial_{q} \varphi(\xi)\right) \prec \sqrt{1+\xi}
$$

If one of the assumptions of Theorem 2 is occurred, then $\varphi \in \mathbb{B}_{q}^{(\beta)}$.

Theorem 3. Let the function $g \in \sqcup$, such that $g(0)=1$ and

$$
1+\xi\left(\partial_{q} g(\xi)\right) \prec \sqrt{1+\xi}, \quad \xi \in \sqcup .
$$

If one of the cases 


$$
\begin{aligned}
& \beta \neq \frac{1}{q+1} ; \\
& \beta \geq-\frac{1.18921 \sqrt{\left(1 /(q+1)^{2}\right)} q+1.18921 \sqrt{\left(1 /(q+1)^{2}\right)}-1}{(q+1)}, \quad 0<q<1, \\
& \beta \leq \frac{1.18921 \sqrt{\left(1 /(q+1)^{2}\right)} q+1.18921 \sqrt{\left(1 /(q+1)^{2}\right)}+1}{(q+1)}, \quad 0<q<1, \\
& \quad|u(\xi)|=\left|\sigma^{2}(\xi)-1\right|<1,
\end{aligned}
$$

holds, then

$$
g(\xi) \prec J_{3}^{(\beta)}(\xi)=\left(1+\xi-\beta \xi^{2}\right) .
$$

Proof. Define a function $\sigma$ as follows:

$$
\sigma(\xi):=1+\xi\left(\partial_{q} g(\xi)\right) .
$$

By the assumption (68) and the meninges of the subordination, we have

$$
1+\xi\left(\partial_{q} g(\xi)\right)=\sqrt{1+u(\xi)}, \quad u(0)=0,|u(\xi)| \leq|\xi|<1,
$$

which yields

$$
u(\xi)=\sigma^{2}(\xi)-1 .
$$

We have to prove that

$$
\begin{aligned}
& \begin{aligned}
\partial_{q}\left[1+u(\xi)-\beta u^{2}(\xi)\right] & =\left(\partial_{q} u(\right. \\
& =\partial_{q}
\end{aligned} \\
& \text { structure, we get } \\
& \left.\beta\left[2 u(\xi)-(1-q) \xi \partial_{q} u(\xi)\right]\right) .
\end{aligned}
$$

Following the above structure, we get

\footnotetext{
$\sigma(\xi)=1+\xi \partial_{q} u(\xi)\left(1-\beta\left[2 u(\xi)-(1-q) \xi \partial_{q} u(\xi)\right]\right)$.
Suppose that there exists a point $\xi_{0} \in \sqcup$, such that
}

for some values $\xi_{0} \in \sqcup$, such that

$$
g(\xi)=\left[1+u(\xi)-\beta u^{2}(\xi)\right] .
$$

Assume not; then, the above conclusion imposes

$$
\sigma(\xi)=1+\xi\left(\partial_{q}\left[1+u(\xi)-\beta u^{2}(\xi)\right]\right) .
$$

By employing the rules of Jackson derivative and the facts

$$
\begin{aligned}
u(q \xi) & =u(\xi)-(1-q) \xi \partial_{q} u(\xi), \\
\partial_{q} u^{2}(\xi) & =\partial_{q} u(\xi)\left[2 u(\xi)-(1-q) \xi \partial_{q} u(\xi)\right],
\end{aligned}
$$

we obtain

We aim to show that 


$$
|u(\xi)|=\left|\sigma^{2}(\xi)-1\right|<1 .
$$

Our method is based on Jack Lemma 1. Assume not. Then, by consuming $u\left(\xi_{0}\right)=e^{i \theta}$, we get

$$
\begin{aligned}
\left|\sigma^{2}\left(\xi_{0}\right)-1\right|= & \left|\left(1+\xi \partial_{q} u(\xi)\left(1-\beta\left[2 u(\xi)-(1-q) \xi \partial_{q} u(\xi)\right]\right)\right)^{2}-1\right|_{\xi=\xi_{0}} \\
& \geq\left|\left(\xi \partial_{q} u(\xi)\left(1-\beta\left[2 u(\xi)-(1-q) \xi \partial_{q} u(\xi)\right]\right)\right)^{2}-1\right|_{\xi=\xi_{0}} \\
= & \left|\left(\xi_{0} \partial_{q} u\left(\xi_{0}\right)\left(1-\beta\left[2 u\left(\xi_{0}\right)-(1-q) \xi_{0} \partial_{q} u\left(\xi_{0}\right)\right]\right)\right)^{2}-1\right| \\
= & \left|\left(\mu e^{i \theta}\left(1-\beta\left[2 e^{i \theta}-(1-q) \mu e^{i \theta}\right]\right)\right)^{2}-1\right| \geq \mathfrak{R}\left(\left(\mu e^{i \theta}\left(1-\beta\left[2 e^{i \theta}-(1-q) \mu e^{i \theta}\right]\right)\right)^{2}-1\right) \\
& =(\mu \cos (\theta)(1-\beta[2 \cos (\theta)-(1-q) \mu \cos (\theta)]))^{2}-1 \geq 1 .
\end{aligned}
$$

Thus, for $\cos (\theta)=1, \mu=1$, the solution of $\left|(\mu \cos (\theta)(1-\beta[2 \cos (\theta)-(1-q) \mu \cos (\theta)]))^{2}-1\right| \geq 1$,

provided one of the following cases:

$$
\checkmark=0, \curlyvee \geq \sqrt{2}, \curlyvee \leq-\sqrt{2},
$$

where

$$
\checkmark:=(1-\beta(1+q))^{2} .
$$

Hence, we obtain one of the following arguments:

$$
\begin{aligned}
& \beta=\frac{1}{q+1} ; \\
& \beta \leq-\frac{1.18921 \sqrt{\left(1 /(q+1)^{2}\right)} q+1.18921 \sqrt{\left(1 /(q+1)^{2}\right)}-1}{(q+1)}, \quad 0<q<1, \\
& \beta \quad \geq \frac{1.18921 \sqrt{\left(1 /(q+1)^{2}\right)} q+1.18921 \sqrt{\left(1 /(q+1)^{2}\right)}+1}{(q+1)}, \quad 0<q<1 .
\end{aligned}
$$

All the above inequalities contradict the assumptions of the theorem, which mean that

$$
g(\xi)<J_{3}^{(\beta)}(\xi)=\left(1+\xi-\beta \xi^{2}\right) .
$$

Corollary 5. Let $\varphi \in \Delta$ be the satisfied subordination:

$$
1+\xi\left(\frac{\xi\left(\partial_{q} \varphi(\xi)\right)}{\varphi(\xi)}\right)<\sqrt{1+\xi} .
$$

If one of the assumptions of Theorem 3 occurred, then $\varphi \in \Delta_{q}^{(\beta)}$.

Proof. Assume

$$
\sigma(\xi)=\left(\frac{\xi \partial_{q} \varphi(\xi)}{\varphi(\xi)}\right) .
$$

Obviously, $\sigma(0)=1$. Thus, according to Theorem 3, we obtain $\varphi \in \Delta_{q}^{(\beta)}$.
Similarly, for $\sigma(\xi)=\partial_{q} \varphi(\xi)$, we have the following consequence.

Corollary 6. Let $\varphi \in \Delta$ be the satisfied subordination:

$$
1+\xi\left(\partial_{q} \varphi(\xi)\right)<\sqrt{1+\xi}
$$

If one of the assumptions of Theorem 3 is occurred, then $\varphi \in \mathbb{B}_{q}^{(\beta)}$.

\section{Conclusion}

From above, we investigate the sufficient conditions to obtain the $q$-subordination of the $q$-starlike class

$$
\left(\frac{\xi \partial_{q} \varphi(\xi)}{\varphi(\xi)}\right)<\downarrow(\xi), \quad \xi \in \sqcup,
$$

where $\mathbb{J}(\xi)=J_{i}^{(\beta)}, i=1,2,3$. Differential inequalities are illustrated, involving the q-differential subordination. Nice geometric presentation is included describing the connected 
Julia functions of different orders. Our class is called 2D parametric subclass of analytic function, and $\beta$ is given in terms of $q$. Note that the case of $1 \mathrm{D}$ parametric subclass is given by

$$
\rrbracket(\xi)=\frac{1+\xi}{1-q \xi} .
$$

It is studied in [21], while null parametric subclass is formulated by

$$
\rrbracket(\xi)=1+\frac{4}{3} \xi+\frac{2}{3} \xi^{2}
$$

and it is investigated in [16].

For future works, one can suggest any types of parametric analytic functions (geometric functions) in the open unit disk. The above $q$-differential subordination formula can be suggested to study the solution of many classes of generalized differential equations such as the class of BriotBouquet differential equation (2).

\section{Data Availability}

No data were used to support this study.

\section{Conflicts of Interest}

The authors declare that they have no conflicts of interest.

\section{Authors' Contributions}

All authors contributed equally and significantly to writing this article and read and agreed to the published version of the manuscript.

\section{Acknowledgments}

This research was supported by Ajman University(2021IRG-HBS-24).

\section{References}

[1] J. E. Littlewood, Lectures on the Theory of Functions, Springer, London, UK, 1944.

[2] S. S. Miller and P. T. Mocanu, Differential Subordinations: Theory and Applications, CRC Press, Boca Raton, FL, USA, 2000.

[3] S. S. Miller, P. T. Mocanu, and M. Reade, "Bazilevic functions and generalized convexity," Revue Roumaine de Mathématique Pures et Appliquées, vol. 19, pp. 213-224, 1974.

[4] M. E. H. Ismail, E. Merkes, and S. David, “"A generalization of starlike functions." Complex Variables," Theory and Application: International Journal, vol. 14, no. 1-4, pp. 77-84, 1990.

[5] S. Agrawal and S. K. Sahoo, "A generalization of starlike functions of order alpha," Hokkaido Mathematical Journal, vol. 46, pp. 15-27, 2017.

[6] H. Srivastava, M. Tahir, B. Khan, Q. Ahmad, and N. Khan, "Some general classes of q-starlike functions associated with the Janowski functions," Symmetry, vol. 11, no. 2, p. 292, 2019.

[7] S. Mahmood, M. Jabeen, H. M. Srivastava, R. Manzoor, and S. M. Riaz, "Some coefficient inequalities of q-starlike functions associated with conic domain defined by q-derivative,"
Journal of Function Spaces, vol. 2018, Article ID 8492072, 13 pages, 2018.

[8] M. Ul-Haq, M. Raza, M. Arif, Q. Khan, and H. Tang, "Qanalogue of differential subordinations," Mathematics, vol. 7, no. 8, p. 724, 2019.

[9] R. W. Ibrahim and B.. Dumitru, "Analytic studies of a class of Langevin differential equations dominated by a class of Julia fractal functions," Kragujevac journal of mathematics, vol. 48, no. 4, pp. 577-590, 2024.

[10] R. W. Ibrahim and D. Baleanu, "Analytic solution of the Langevin differential equations dominated by a multibrot fractal set," Fractal and Fractional, vol. 5, no. 2, p. 50, 2021.

[11] Z. E. Abdulnaby, R. W. Ibrahim, and R. W. Ibrahim, "On a subclass of analytic functions of fractal power with negative coefficients," Series Iii-Matematics, Informatics, Physics, vol. 13 , no. 2, pp. 387-398, 2020.

[12] W. C. Ma and D. Minda, "A unified treatment of some special classes of univalent functions," in Proceedings of the Conference on Complex Analysis, Z. Li, F. Ren, L. Yang, and S. Zhang, Eds., International Press, Cambridge, MA, USA, January 1992.

[13] F. H. Jackson, "XI-On q-functions and a certain difference operator," Earth and Environmental Science Transactions of the Royal Society of Edinburgh, vol. 46, no. 2, pp. 253-281, 1909.

[14] D. O. Jackson, T. Fukuda, O. Dunn, and E. Majors, "On q-definite integrals," The Quarterly Journal of Pure and Applied Mathematics, 1910.

[15] T. M. Seoudy and M. K. Aouf, "Coefficient estimates of new classes of q-starlike and q-convex functions of complex order," Journal of Mathematical Inequalities, vol. 10, no. 1, pp. 135-145, 2016.

[16] S. Zainab, A. Shakeel, M. Imran, N. Muhammad, H. Naz, and M. Arif, "Sufficiency criteria for-starlike functions associated with cardioid," Journal of Function Spaces, vol. 2021, Article ID 9999213, 9 pages, 2021.

[17] R. W. Ibrahim, R. M. Elobaid, and S. J. Obaiys, "Geometric inequalities via a symmetric differential operator defined by quantum calculus in the open unit disk," Journal of Function Spaces, vol. 2020, Article ID 6932739, 8 pages, 2020.

[18] R. W. Ibrahim, R. M. Elobaid, and S. J. Obaiys, "A class of quantum Briot-Bouquet differential equations with complex coefficients," Mathematics, vol. 8, no. 5, p. 794, 2020.

[19] R. W. Ibrahim and B. Dumitru, "On quantum hybrid fractional conformable differential and integral operators in a complex domain," Revista de la Real Academia de Ciencias Exactas, Fisicas y Naturales. Serie A. Matematicas, vol. 115, pp. 1-13, 2021.

[20] R. W. Ibrahim, "Geometric process solving a class of analytic functions using q-convolution differential operator," Journal of Taibah University for Science, vol. 14, no. 1, pp. 670-677, 2020.

[21] A. Cetinkaya and P. G. Yasar, " $q$-Harmonic mappings for which analytic part is q-convex functions of complex order," Hacettepe Journal of Mathematics and Statistics, vol. 47, no. 4, pp. 813-820, 2018. 